\title{
Tuning the coupling of a single quantum dot to a photonic crystal waveguide
}

\author{
Nielsen, Henri Thyrrestrup; Lodahl, Peter; Lund-Hansen, Toke
}

Published in:

Conference on Lasers and Electro-Optics, 2009 and 2009 Conference on Quantum electronics and Laser Science Conference

Publication date:

2009

Document Version

Publisher's PDF, also known as Version of record

Link back to DTU Orbit

Citation (APA):

Nielsen, H. T., Lodahl, P., \& Lund-Hansen, T. (2009). Tuning the coupling of a single quantum dot to a photonic crystal waveguide. In Conference on Lasers and Electro-Optics, 2009 and 2009 Conference on Quantum electronics and Laser Science Conference: CLEO/QELS 2009 (pp. 1-2). IEEE.

\section{General rights}

Copyright and moral rights for the publications made accessible in the public portal are retained by the authors and/or other copyright owners and it is a condition of accessing publications that users recognise and abide by the legal requirements associated with these rights.

- Users may download and print one copy of any publication from the public portal for the purpose of private study or research.

- You may not further distribute the material or use it for any profit-making activity or commercial gain

- You may freely distribute the URL identifying the publication in the public portal 


\title{
Tuning the Coupling of a Single Quantum Dot to a Photonic Crystal Waveguide
}

\author{
H. Thyrrestrup, T. Lund-Hansen, P. Lodahl \\ DTU Fotonik, Department of Photonics Engineering, Technical University of Denmark, Ørsteds Plads 343, DK-2800 Kgs. Lyngby, \\ Denmark \\ E-mail:htni@fotonik.dtu.dk \\ Abstract: We present time-resolved spontaneous emission measurements of a single quantum dot that is \\ temperature tuned around the band edge of a photonic crystal waveguide. $85 \%$ efficient coupling to the slow- \\ light waveguide mode is obtained. \\ (C)2008 Optical Society of America \\ OCIS codes: (270.5580) Quantum electrodynamics; (230.5590) Quantum-well, -wire and -dot devices.
}

\section{Efficient single-photon sources by coupling quantum dots to nanostructures}

A highly efficient single-photon source is a key component in many quantum communication protocols and will allow implementing linear optics quantum computing. An efficient single-photon source can be obtained by harvesting the emitted photons from a quantum dot into a single optical mode. A key requirement is the efficient coupling to this mode. Photonic crystal nanocavities have successfully been used to demonstrate a high coupling of photons from a single quantum dot to the localized cavity mode [1]. One draw back of this approach, however, is that the photons need to be coupled out of the cavity for further processing, which reduces the overall efficiency of the device. Furthermore, the cavity only exhibits high collection efficiency in a narrow bandwidth that is inversely proportional to the coupling strength.

One proposed approach to overcome these limitations is to use photonic crystal waveguides, in which the photons from the quantum dot are coupled directly into the wide-bandwidth propagating mode $[2,3]$. The coupling is strongly enhanced near the band edge of the photonic crystal waveguide due to the significant slow-down of light. We have previously demonstrated the feasibility of this approach using self-assembled quantum dots, and by averaging over an ensemble of single quantum dot emitters, we extracted a coupling efficiency to the waveguide mode approaching 90\% [4]. Here we extend this work and temperature tune a single quantum dot around the band edge of a photonic crystal waveguide while measuring the spontaneous emission decay rate. In this way the coupling efficiency of the quantum dot to the waveguide can be extracted directly and related to the precise frequency variation of the photonic crystal waveguide mode in the slow-light regime.

2. Experimental demonstration of tuning of the coupling between a single quantum dot and the photonic crystal waveguide

We study 2D photonic crystals consisting of a $150 \mathrm{~nm}$ thick GaAs membrane where the waveguide is formed by leaving out a single row of holes. The waveguide is $100 \mu \mathrm{m}$ long and centered in a $7 \mu \mathrm{m}$ wide photonic crystal with a lattice parameter of $a=256 \mathrm{~nm}$ and a hole radius of $r=0.299 \mathrm{a}$. The center of the membrane contains a single layer of self-assembled InAs quantum dots characterized by a center emission wavelength of $950 \mathrm{~nm}$, an inhomogeneous broadening of $50 \mathrm{~nm}$, and a decay rate in bulk GaAs of $\sim 1 \mathrm{~ns}^{-1}$. Each quantum dot acts as a single-photon emitter in the weak pumping regime.

The quantum dots are pumped with a pulsed Ti:Sapphire laser at $850 \mathrm{~nm}$ corresponding to excitation in the wetting layer. The spontaneously emitted photons are collected using a NA=0.6 microscope objective and directed to a single mode fiber. It acts as a pinhole in a confocal setup, which gives a spatial resolution of $\sim 1.5 \mu \mathrm{m}$ (FWHM). Emission spectra are recorded with a spectrometer equipped with a CCD camera, and time-resolved measurements are performed with an APD. The resolution of the CCD spectra is $0.05 \mathrm{~nm}$, and the wavelength interval sent through to the APD is $0.1 \mathrm{~nm}$. The time resolution of the APD is $280 \mathrm{ps}$, as obtained from the FWHM of the instrument response function.

The experiment is done by first aligning the collection spot precisely on top of the waveguide and then selecting a single quantum dot line that is efficiently coupled to the waveguide at $10 \mathrm{~K}$. The efficient coupling is identified by recording the spontaneous emission decay curve since coupled quantum dots decay fast due to the Purcell effect. An example is shown as the blue curve in fig. 1(a). The quantum dot emission wavelength is subsequently tuned towards the steep slope of the waveguide band edge, by changing the temperature from $10 \mathrm{~K}$ to $60 \mathrm{~K}$ in intervals of 


\section{IThB6.pdf}
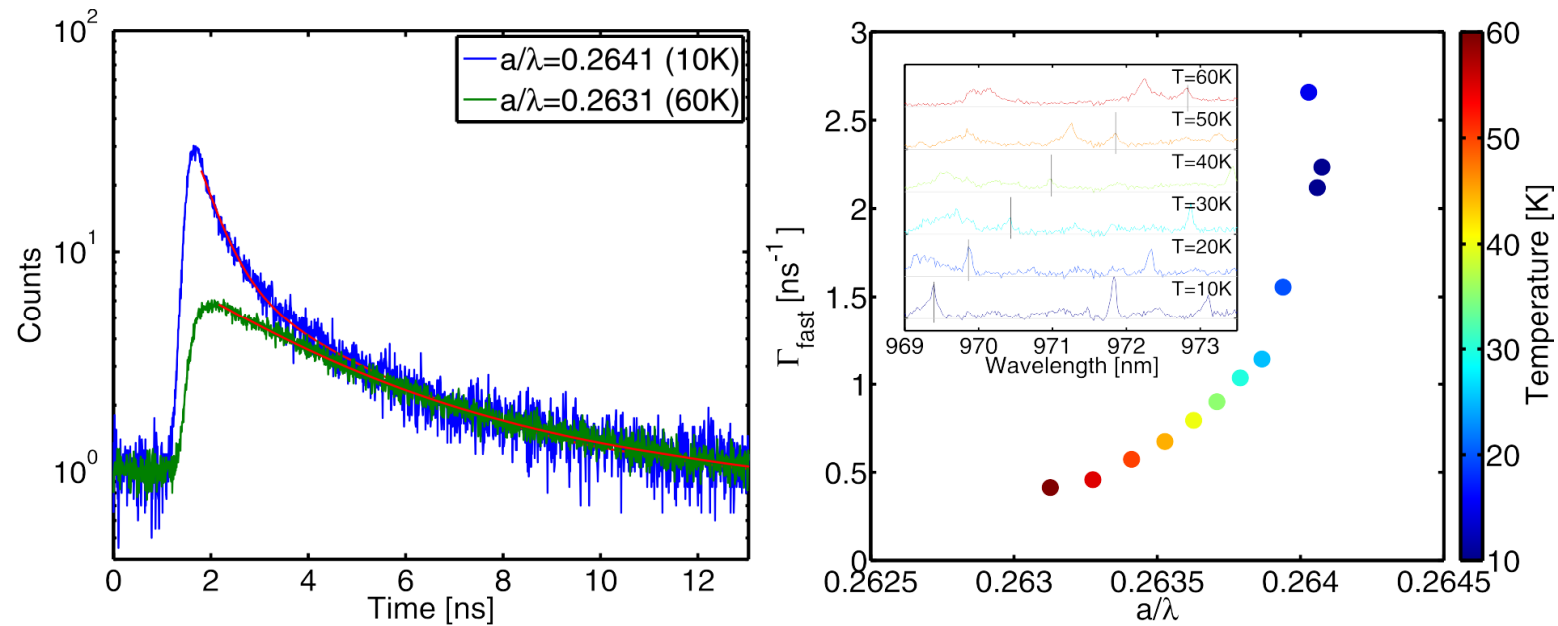

Figure 1: (a) Two decay curves for the same quantum dot at $10 \mathrm{~K}$ (blue) and $60 \mathrm{~K}$ (green), respectively. (b) Fast decay rate of a single quantum dot for varying emission wavelength $\lambda$ near the waveguide band edge. The corresponding temperatures are marked with colors. (Inset) Quantum dot emission spectra at six different temperatures. The position of the coupled quantum dot is marked by a black line.

$5 \mathrm{~K}$. At each temperature, a decay curve and a corresponding CCD spectrum is obtained. Six examples of emission spectra are shown in the inset of fig. 1(b) with the selected quantum dot marked by a vertical black line. Using this method we can reproducibly tune the quantum dot over $3.5 \mathrm{~nm}$.

The decay curves at $10 \mathrm{~K}$ (blue) and $60 \mathrm{~K}$ (green) are shown in fig. 1(a) and we observe a pronounced difference in the fast decay rate, corresponding to an altered coupling between the waveguide and the quantum dot. At $10 \mathrm{~K}$ the quantum dot couples efficiently to the waveguide mode leading to an accelerated decay, whereas at $60 \mathrm{~K}$ the decay is slow due to the suppressed local density of stated in the 2D photonic crystal bandgap. The red lines are fits to the data applying a bi-exponential model convoluted with the APD instrument function. The fast decay rate is dominated by the radiative decay rate, which is strongly enhanced due to the coupling to the waveguide mode, and contains an additional non-radiative part. The slow decay rate is dominated by non-radiative recombination of dark excitons, and will not be discussed further here.

The fitted decay rates are plotted as a function of reduced frequency $(\mathrm{a} / \lambda)$ and colored with temperature in fig. 2(b). We see a clear enhanced decay rate around $\mathrm{a} / \lambda=0.264$ with a maximum of $\Gamma_{1}=2.66 \mathrm{~ns}^{-1}$, which corresponds to the band edge of the waveguide mode. For lower frequencies, the decay rates rapidly decrease to $\Gamma_{2}=0.41 \mathrm{~ns}^{-1}$, which is inhibited due to the photonic crystal.

From the measurements we can extract the $\beta$-factor, which is the figure-of-merit for a single-photon source and expresses the fraction of the emitted photons that are coupled to the waveguide. It is given by

$$
\beta=\frac{\Gamma_{W G}}{\Gamma_{W G}+\Gamma_{\text {rad }}+\Gamma_{\text {nrad }}},
$$

where $\Gamma_{\mathrm{WG}}$ is the coupling rate to the waveguide, $\Gamma_{\text {rad }}$ is the coupling rate to all other radiation modes, and $\Gamma_{\text {nrad }}$ is the non-radiative decay rate. Assuming that $\Gamma_{1}$ reflects a situation where the coupling to the waveguide is negligible, we have, $\Gamma_{\text {rad }}+\Gamma_{\text {nrad }}=\Gamma_{2}$ and $\Gamma_{\mathrm{WG}}=\Gamma_{1}-\Gamma_{2}$ leading to $\beta=85 \%$. This very high efficiency illustrates the great potential of a photonic crystal waveguide for single-photon source applications, and makes it a very promising candidate for novel nonlinear quantum optics experiments at the few-photon level [5].

\section{References}

[1] K. Hennessy et al., Nature (London) 445, 896 (2007)

[2] G. Lecamp, P. Lalanne, and J.P. Hugonin, Phys. Rev. Lett. 99, 023902 (2007).

[3] V.S.C. Manga Rao, and S. Hughes, Phys Rev. Lett. 99, 193901 (2007).

[4] T. Lund-Hansen, S. Stobbe, B. Julsgaard, H. Thyrrestrup, T. Sunner, M. Kamp, A. Forchel, and P. Lodahl, Phys. Rev. Lett. 101, 113903 (2008).

[5] D.E. Chang, A.S. Sørensen, E.A. Demler, and M.D. Lukin, Nature Physics 3, 807 (2007). 